\title{
GALAXY CLUSTERS IN THE IRAC DARK FIELD. II. MID-INFRARED SOURCES
}

\author{
J. E. Krick ${ }^{1}$, J. A. Surace ${ }^{1}$, D. Thompson ${ }^{2}$, M. L. N. Ashby ${ }^{3}$, J. L. Hora ${ }^{3}$, V. GorJian ${ }^{4}$, And L. Yan ${ }^{1}$ \\ ${ }^{1}$ Spitzer Science Center, MS 220-6, California Institute of Technology, Jet Propulsion Laboratory, Pasadena, CA 91125, USA; jkrick@ caltech.edu \\ ${ }^{2}$ Large Binocular Telescope Observatory, University of Arizona, Tucson, AZ 85721, USA \\ ${ }^{3}$ Harvard-Smithsonian Center for Astrophysics, 60 Garden Street, Cambridge, MA 02138, USA \\ ${ }^{4}$ Jet Propulsion Laboratory, California Institute of Technology, Pasadena, CA 91109, USA \\ Received 2008 December 17; accepted 2009 May 14; published 2009 June 30
}

\begin{abstract}
We present infrared (IR) luminosities, star formation rates (SFR), colors, morphologies, locations, and active galactic nuclei (AGNs) properties of $24 \mu \mathrm{m}$ detected sources in photometrically detected high-redshift clusters in order to understand the impact of environment on star formation (SF) and AGN evolution in cluster galaxies. We use three newly identified $z=1$ clusters selected from the IRAC dark field; the deepest ever mid-IR survey with accompanying, 14 band multiwavelength data including deep Hubble Space Telescope imaging and deep wide-area Spitzer MIPS $24 \mu \mathrm{m}$ imaging. We find 90 cluster members with MIPS detections within two virial radii of the cluster centers, of which 17 appear to have spectral energy distributions dominated by AGNs and the rest dominated by SF. We find that $43 \%$ of the star-forming sample have IR luminosities $L_{\mathrm{IR}}>10^{11} L_{\odot}$ (luminous IR galaxies). The majority of sources (81\%) are spirals or irregulars. A large fraction (at least $25 \%$ ) show obvious signs of interactions. The MIPS-detected member galaxies have varied spatial distributions as compared to the MIPS-undetected members with one of the three clusters showing SF galaxies being preferentially located on the cluster outskirts, while the other two clusters show no such trend. Both the AGN fraction and the summed SFR of cluster galaxies increase from redshift zero to one, at a rate that is a few times faster in clusters than over the same redshift range in the field. Cluster environment does have an effect on the evolution of both AGN fraction and SFR from redshift one to the present, but does not affect the IR luminosities or morphologies of the MIPS sample. SF happens in the same way regardless of environment making MIPS sources look the same in the cluster and field, however the cluster environment does encourage a more rapid evolution with time as compared to the field.
\end{abstract}

Key words: cosmology: observations - galaxies: active - galaxies: clusters: general - galaxies: evolution galaxies: photometry - infrared: galaxies

\section{INTRODUCTION}

Galaxy groups and clusters represent the dense environments required for hierarchical galaxy formation. Cluster galaxies potentially follow a different evolutionary path from isolated field galaxies because of a cluster's large gravitational potential and hot, X-ray emitting gas. As galaxy clusters form and grow throughout time by infall of galaxies and groups of galaxies, the simple picture is one of the member galaxies that are transformed from blue, late types with signs of star formation (SF) to red, early types with no SF. This conversion most likely happens through a combination of processes including mergers, SF bursts, ram pressure stripping, and harassment (van Dokkum 2005; Gunn \& Gott 1972; Moore et al. 1996).

This work comes at a key time in the study of star-forming galaxies and active galactic nuclei (AGNs) in high-redshift clusters. Only recently have we been able to study SF in clusters at $z=1$. There are only a few well studied clusters at $z=1$, although the number is growing rapidly and will continue to do so with upcoming Sunyaev-Zeldovich and large sky surveys (Staniszewski et al. 2008). Additionally, traditional measures of SF are difficult to obtain at high redshifts. $\mathrm{H} \alpha$ shifts out of the optical band by $z \sim 0.5$. Both $\mathrm{O}$ II and $\mathrm{H} \alpha$ narrow band surveys with specially designed filters (Poggianti et al. 2008; Finn et al. 2008) are possible, but optical emission-line spectroscopy at high redshift is telescope time intensive, and narrowband surveys only work for the designed redshift. Both of these measures are also affected by dust obscuration. However, with Spitzer MIPS we are able to measure obscured SF at large redshifts with relative ease.
That we see SF in galaxy clusters at all is worth investigation. $\mathrm{O}$ and $\mathrm{B}$ stars live for less than 10 million years, so a single, triggered episode of SF is likely to last for less than a few tens of million years. If the infall time of a galaxy into the center of a cluster is roughly $1 \mathrm{Gyr}$ (assuming $1 \mathrm{Mpc}$ radius and $1000 \mathrm{~km} \mathrm{~s}^{-1}$ velocities) and all galaxies somehow have their SF suppressed upon entering the cluster environment, we would expect to see no SF in the centers of clusters, unless it is triggered, in situ, by mergers. We would therefore expect to see no blue, isolated galaxies with heightened SF in the central regions of clusters. Furthermore, if SF is actually first triggered and then suppressed upon cluster entry, as it has been suggested processes like ram pressure stripping could do (Bekki \& Couch 2003; Kronberger et al. 2008), then we should see SF in isolated spirals on the outskirts of the clusters. Based on this timescales argument we should potentially see SF on the edges of clusters, but not in the centers, unless it is merger driven. SF triggered by galaxy interactions and mergers is not dependent on cluster environment, instead on the relative velocities of the galaxies. As such this form of SF can occur anywhere in the cluster environment or the field, and is more likely to happen in lower mass clusters or groups due to the lower relative velocities.

There is intriguing evidence that star formation rates (SFRs) in clusters increase with redshift out to at least $z=0.83$ (Bai et al. 2007). We investigate if this evolution follows that in the field, implying that cluster environment does not impact SF. We examine this claim by increasing the number of clusters studied at high redshift and extending the redshift range out to redshift one. There are only two clusters with published MIPS $24 \mu \mathrm{m}$ SFRs at redshifts above 0.8, both at $z=0.83$ (MS1054-03, RX J0152 Bai et al. 2007; Marcillac et al. 2007; Saintonge et al. 
2008). Koyama et al. (2008) use the Infrared Camera on Akari (Onaka et al. 2007; Murakami et al. 2007) at $15 \mu \mathrm{m}$ s to study a redshift 0.81 cluster. Although this is a mid-IR measurement of SFR, they use a different rest-frame wavelength to convert to $L_{\mathrm{IR}}$ which carries a different set of assumptions. Our survey is unique in that we double the number of published high-redshift clusters with $24 \mu \mathrm{m}$ SFRs by adding a large-scale structure at $z=1$ containing three clusters/groups with larger number statistics and deeper $L_{\mathrm{IR}}$ measurements over a large area.

In addition to SF, we examine for the first time MIPSdetected AGNs in cluster environments as a different line of evidence of galaxy activity. The same processes which affect $\mathrm{SF}$ in galaxies will also effect the AGN on roughly the same timescales (Hopkins et al. 2008). AGNs and SF are linked not only because they both require a cold gas reservoir to ignite, but also due to both SF an AGN feedback mechanisms which have the ability to destroy or remove the cold gas and halt either the SF, the AGN activity, or both (Croton et al. 2006). AGNs can put a halt to SF by blowing out or heating the gas, and similarly SF can use up gas thereby removing the source for a central engine. We expect the AGN fraction at high redshift to be higher than at low redshift in clusters based on evidence both in clusters and the field (Osmer 2004; Eastman et al. 2007; Kocevski et al. 2008; Galametz et al. 2009). We examine if the AGN fraction in clusters tracks the redshift evolution of that in the field or is enhanced by the cluster environment.

This paper is structured in the following manner. In Sections 2 and 3 we discuss the data and derived photometric redshift determination. Details of the sample selection are presented in Section 4. In Section 5, we present the AGN fraction, infrared (IR) luminosities, SFRs, colors, morphologies, and radial distributions of both the star-forming and AGN samples. The paper is summarized and conclusions are drawn in Section 6. Throughout this paper we use $H_{0}=70 \mathrm{~km} \mathrm{~s}^{-1} \mathrm{Mpc}^{-1}, \Omega_{M}=0.3, \Omega_{\Lambda}$ $=0.7$. With this cosmology, the luminosity distance at $z=1$ is $6607 \mathrm{Mpc}$, but the angular diameter distance is a factor of $(1+z)^{2}$ less, or $1652 \mathrm{Mpc}$. All photometry is quoted in the AB magnitude system.

\section{OBSERVATIONS AND DATA REDUCTION}

\subsection{The IRAC Dark Field}

The survey region is the IRAC Dark Field, centered at approximately $17 \mathrm{~h} 40 \mathrm{~m}+69 \mathrm{~d}$. The field is located a few degrees from the north ecliptic pole (NEP) in a region that is darker than the actual pole and is in the Spitzer continuous viewing zone so that it can be observed any time IRAC is powered on for observing. These observing periods are called instrument "campaigns," and occur roughly once every three to four weeks and last for about a week. Sets of long exposure frames are taken on the Dark Field at least twice during each campaign totaling roughly $4 \mathrm{hr}$ of integration time per campaign, and these data are used to derive dark current/bias frames for each channel. The dark frames are used by the pipeline in a manner similar to "median sky" calibrations as taken in ground-based near-IR observing to produce the Basic Calibrated Data (BCD) for all science observations. Each set of dark calibration observations collects roughly $2 \mathrm{hr}$ of integration time at the longest exposure times in each channel.

The resulting observations are unique in several ways. The Dark Field lies near the lowest possible region of zodiacal background, the primary contributor to the IR background at these wavelengths, and as such is in the region where the greatest sensitivity can be achieved in the least amount of time. The area was also chosen specifically to be free of bright stars and very extended galaxies, which allows clean imaging to very great depth. The observations are done at many position angles (which are a function of time of observation) leading to a more uniform final point-spread function (PSF). Finally, because the calibration data are taken directly after anneals, they are more free of artifacts than ordinary guest observer (GO) observations. Over the course of the mission, the observations have filled in a region $20^{\prime}$ in diameter with a total of $\sim 350 \mathrm{hr}$ devoted to the project; $\sim 70 \mathrm{hr}$ pixel $^{-1}$ in the center of each band as of the writing of this paper. This will create the deepest midIR survey, exceeding the depth of the deepest planned regular Spitzer surveys over several times their area. Furthermore, this is the only field for which a 5+year baseline of mid-IR periodic observations is expected.

The IRAC data are complemented by imaging data in 14 other bands with facilities including Palomar, MMT, Hubble Space Telescope (HST), Akari, Spitzer MIPS, and Chandra ACIS-I as well as Palomar optical spectroscopy. Although the entire dark field is $>20^{\prime}$ in diameter, because of spacecraft dynamics the central $\sim 15^{\prime}$ is significantly deeper and freer of artifacts. Therefore, it is this area which we have matched with the additional observations. The entire data set will be presented in detail in a future paper (Krick et al. 2009). For completeness we briefly discuss here the Spitzer IRAC, Spitzer MIPS, HST ACS, and Palomar optical spectroscopy as they are the most critical to this work. All space-based data sets are publicly available through their respective archives.

\subsection{Spitzer IRAC}

This work is based on a preliminary combination of $75 \mathrm{hr}$ of IRAC imaging, which is $\approx 30 \%$ of the expected depth not including the warm mission. The BCD product produced by the Spitzer Science Center was further reduced using a modified version of the pipeline developed for the SWIRE survey (Surace et al. 2005). This pipeline primarily corrects image artifacts and forces the images onto a constant background (necessitated by the continuously changing zodiacal background as seen from Spitzer). The data were co-added onto a regularized 0.'6 grid using the MOPEX software developed by the Spitzer Science Center.

Experiments with DAOPHOT demonstrate that nearly all extragalactic sources are marginally resolved by IRAC, particularly at the shorter wavelengths, and hence point-source fitting is inappropriate. Instead, photometry is done using the high spatial resolution ACS data as priors for determining the appropriate aperture shape for extracting the Spitzer data. We do this by first running source detection and photometric extraction on the coadded IRAC images using a matched filter algorithm with image backgrounds determined using the mesh background estimator in SExtractor (Bertin \& Arnouts 1996). This catalog is merged with the HST ACS catalog. For every object in that catalog, if the object is detected in ACS then we use the ACS shape parameters to determine the elliptical aperture size for the IRAC images. ACS shape parameters are determined by SExtractor on isophotal object profiles after deblending, such that each ACS pixel can only be assigned to one object (or the background). For objects which are not detected in ACS, but which are detected in IRAC, we simply use the original IRAC SExtractor photometry. Because of the larger IRAC beam, we impose a minimum semimajor axis radius of $2^{\prime \prime}$. In all cases aperture corrections are computed individually from PSFs provided by the Spitzer 
Science Center based on the aperture sizes and shapes used for photometry.

Final aperture photometry was performed using custom extraction software written in IDL and based on the APER and MASK_ELLIPSE routines with the shape information from SExtractor, from either ACS or IRAC as described above, using local backgrounds. Because we use local backgrounds, the measured fluxes of objects near the confusion limit should have a larger scatter than those nonconfused objects, but will on average be the correct flux. This will not effect the photometric redshifts, as it will likely shift all IRAC points up or down, but not relative to each other.

Determining the detection limits of the IRAC data is complicated by varying exposure times across the field, source confusion, and our use of ACS locations as priors for photometry. Because of these three complexities, there is not one single value for the detection limit of the survey, however this work is limited by the MIPS detection limits and not IRAC or ACS. We measure nominal $95 \%$ completeness limits in the IRAC passbands from a number count diagram at $3.6,4.5,5.8$, and $8.0 \mu \mathrm{m}$ to be 0.2 , $0.17,0.11$, and $0.11 \mu \mathrm{Jy}$, respectively.

\subsection{Spitzer MIPS24}

The Spitzer MIPS $24 \mu \mathrm{m}$ data were taken in large-field photometry mode with a $30 \mathrm{~s}$ exposure time. A $3 \times 3$ MIPS field-of-view grid was mapped and repeated five times, with multiple dithers and chops totaling $224 \operatorname{arcmin}^{2}$ in the center of the IRAC image. There were a total of 1080 separate exposures with a final total depth of 60 minutes per pointing on the sky. The MIPS data were processed by the Spitzer Science Center into individual image BCDs. However, substantial "jailbar" artifacts, as well as a significant gradient, were visible. All of the frames were forced to a common background by applying an additive constant to the entire frame. A "delta-dark" was then generated from the median of all frames; the great degree of dithering in the data allows this process to reject all actual celestial objects in the frames from the median stack. That stack was then adjusted to a median overall zero value, and then subtracted from all the data. It currently is not known whether the gradient effect is additive or multiplicative, although our experience with other $\mathrm{Si}$ :As arrays of this kind strongly suggests (from a physical basis) that it is additive. However, we reduced the data both ways, and found no difference at any detectable level. The data were then co-added using the MOPEX software package onto exactly the same projection system as used for IRAC, albeit with $1^{\prime \prime} .2$ pixels.

IRAF DAOFIND was used for object detection. We supply the code with the PSF FWHM and background sigma values taken by examining the image. DAOFIND then counts the flux within an annulus of diameter FWHM and flags any set of pixels as a detection where that flux is above a threshold of $5 \sigma$. To deal with confused sources, we perform object detection iteratively. After the first run through DAOFIND, all objects are subtracted from the image using a PSF determined from the detected objects. DAOFIND is then re-run on the residual image. To ensure that the iterative detection is not dominated by noise, we manually check all detections within the cluster area by eye (see Section 4). With the exception of a handful of galaxies, all MIPS detections appear as point sources. Photometry on all detected sources is done with the IRAF task ALLSTAR which fits PSFs to groups of objects simultaneously. An aperture correction of 1.4 is applied for flux beyond the 6.5 pixel radius at which the PSF star was normalized. This correction factor is calculated from a curve of growth based on the composite PSF star. Using this method the $3 \sigma$ detection limit is $17.3 \mu \mathrm{Jy}$. These noise properties are comparable to the GOODS slightly longer exposure (77 minutes) data set that has a $3 \sigma$ limit of $12 \mu \mathrm{Jy}$.

\subsection{HST ACS}

The HST observations consist of 50 orbits with the ACS comprising 25 separate pointings, all with the F814W filter (observed $I$ band). Within each pointing eight dithered images were taken for cosmic-ray rejection and to cover the gap between the two ACS CCDs. The ACS pipeline CALACS was used for basic reduction of the images. Special attention was paid to bias subtraction, image registration, and mosaicing. Pipeline bias subtraction was insufficient because it does not measure the bias level individually from each of the four amplifiers used by ACS. We make this correction ourselves by subtracting the mean value of the best-fit Gaussian to the background distribution in each quadrant. Due to distortions in the images, registration and mosaicing was performed with a combination of IRAF's TWEAKSHIFTS, MULTIDRIZZLE, and SWARP version 2.16.0 from Terapix. The actual task of mosaicing the final image was complicated by the large image sizes. The single combined mosaic image is $1.7 \mathrm{~GB}$ and reading in all 200 images $(160 \mathrm{Mb}$ each) for combination is impossible for most software packages.

The final combined ACS image is $\sim 15^{\prime}$ diameter coincident with the deepest part of the IRAC Dark Field and is made with the native 0 .'05 per pixel resolution. Photometry was performed in a standard manner with SEXTRACTOR. The $3 \sigma$ detection limit for point sources is $F 814 W=28.6(A B)$.

\subsection{Palomar Optical Spectroscopy}

The Palomar data consists of a total of four nights at the Hale 200" telescope with the COSMIC spectrograph. COSMIC, at prime focus, has a 13.6 field of view, and 0.'4 pixels. Observations were made on a total of four photometric nights in June of 2007 and 2008 with the $3001 \mathrm{~mm}^{-1}$ grating with a dispersion of $2 \AA$ per pixel. We chose a slit width of $1^{\prime \prime} .5$ to match our $1^{\prime \prime}-1$ ". 5 seeing. The optical band covered by this instrument includes such spectral features as $\mathrm{CaH} \& \mathrm{~K}$, [O II], [O III], $\mathrm{H} \alpha$, $\mathrm{H} \beta, \mathrm{H} \delta, \mathrm{G}$ band, and the $4000 \AA$ break. During both runs we were able to observe a total of 11 slit masks of $\sim 25$ galaxies each with exposure times of on average 80 minutes divided into multiple exposures. One $\mathrm{Hg}-\mathrm{Ar}$ lamp and one flat were taken through each mask at the beginning of the night for calibration. Galaxies were chosen to be brighter than $r=21(\mathrm{AB})$ with priority given to those with MIPS 24 or $70 \mu \mathrm{m}$ detections to boost the chance of seeing an emission line and thereby getting a secure redshift.

Reduction was done with IRAF mainly through the BoGUS2006 ${ }^{5}$ scripts. Prior to running bogus, images were overscan and bias subtracted. Bogus itself does a two-dimensional reduction including flat-fielding, cosmic-ray removal, sky subtraction, fringe suppression, and combination of frames. The same reduction is performed on both science images and arcs. The standard IRAF tasks of APALL, IDENTIFY, and DISPCOR were used to wavelength correct, trace, and extract the spectra with a secondary background subtraction for minor level changes. Onedimensional spectra were extracted for a total of 200 galaxies with measurable continuum.

\footnotetext{
5 https://zwolfkinder.jpl.nasa.gov/stern/homepage/bogus.html
} 
No single cluster galaxy was bright enough to have a spectrum observed at Palomar. Instead these spectra are used to calibrate our photometric redshifts.

\section{PHOTOMETRIC REDSHIFTS}

The combined IRAC and ACS catalog contains over 50,000 objects which makes acquisition of spectroscopic redshifts impractical. Even confirmation spectroscopy of red galaxies at $z=1$ in our three candidate clusters will require many nights on 8-10 m class telescopes and is therefore also impractical. In lieu of spectroscopy we use our extensive multiwavelength, broadband catalog to build spectral energy distributions (SEDs) using up to 13 bands $\left(u^{\prime}, g^{\prime}, r^{\prime}, i^{\prime}, \mathrm{F} 814 \mathrm{~W}, z^{\prime}, J, H, K, 3.6\right.$, $4.5,5.8$, and $8.0 \mu \mathrm{m}$ ) from which we derive photometric redshifts. A full discussion of the accuracy of photometric redshift determinations is beyond the scope of this paper (but see, for example, Mobasher et al. 2004; Brodwin et al. 2006; Bolzonella et al. 2000).

These SEDs are fitted with template spectra derived from galaxies in the Spitzer wide area IR survey (SWIRE; Polletta et al. 2007). These templates have been used successfully by a number of surveys at a range of redshifts for all galaxy types (Adami et al. 2008; Negrello et al. 2009; Salvato et al. 2009; Ilbert et al. 2009). Since the SWIRE templates are based on Spitzer observations we find them the best choice to use as models for this data set. We use 15 templates including ellipticals, spirals, star-forming galaxies, and AGN. Photometric redshifts are calculated using HYPERZ; a chisquared minimization fitting program including a correction for interstellar reddening (Bolzonella et al. 2000; Calzetti et al. 2000).

Errors in photometric redshifts are determined by comparing the photometric redshifts with spectroscopic redshifts. Spectroscopic redshifts were determined using both IRAF tasks EMSAO and XCSAO. Specifically EMSAO searches the spectrum for both absorption and emission lines which it correlates with a given line list. XCSAO cross-correlates the spectrum with known galaxy templates which allows us to use features like the $4000 \AA$ break and the rest of the spectral shape to identify redshifts. Both techniques were used together to arrive at the best-fit redshift for each galaxy. We used 17 spectral templates of galaxies and AGN from the compilation of the HST Calibration Database System (Francis et al. 1991; Kinney et al. 1996; Calzetti et al. 1994). We applied a very strict requirement that all emission and absorption features in the one-dimensional spectra were confirmed by eye on the two-dimensional spectra and that multiple lines be identified in all cases to avoid incorrect redshift determination due to cosmic rays or noise features from sky line subtraction.

We were able to successfully determine redshifts for 87 galaxies. This represents a conservative sample of "good" redshift determinations defined to have either high signal-tonoise emission lines or multiple absorption lines and good crosscorrelations. We then compare the spectroscopic to photometric redshifts to quantify the error on the latter (Figure 1). There are cases where HyPERz has failed to fit the correct redshift which is obvious when looking at the SED fit. Those galaxies, as characterized by a $\chi^{2}$ value greater than 50 , are not included in this comparison or the cluster sample below. The error on the photometric redshifts is $0.064(1+z)$. Note that this error is quoted as a function of redshift and so takes into account the increasing scatter with redshift. This accuracy is similar to other IRAC based multiwavelength studies (Brodwin et al. 2006;

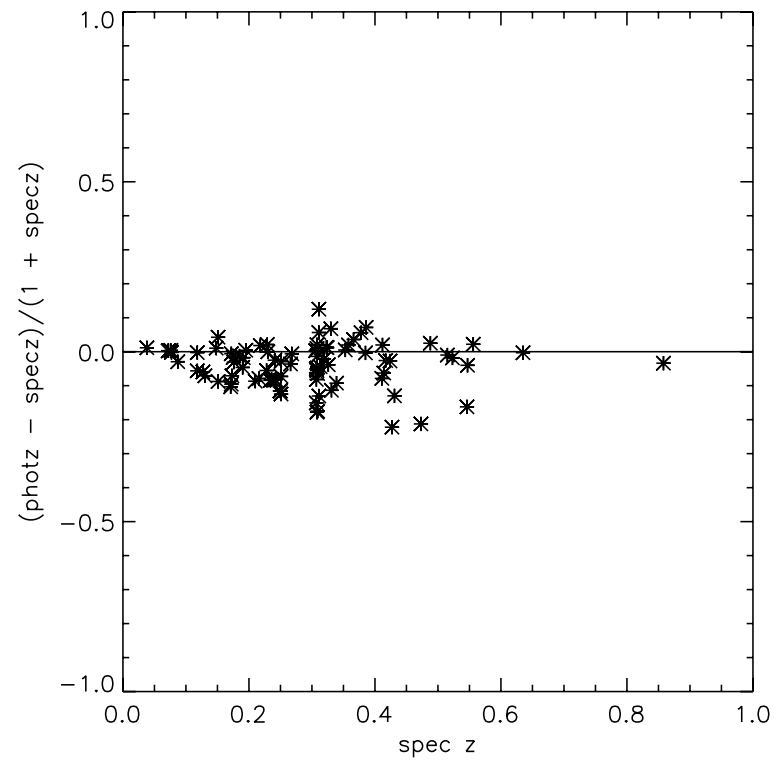

Figure 1. Comparison of spectroscopically and photometrically determined redshifts. The scatter implies an error on the photometric redshifts of $0.064(1+z)$

Rowan-Robinson et al. 2008). We are confident that our quoted accuracy will hold in extrapolating our photometric redshifts out to $z=1$ because at that redshift the Balmer break is shifted into our HST ACS F814W and MMT $z^{\prime}$ which are our most sensitive bands. Secondly the peak of the stellar distribution is shifted into the IRAC bands where we have excellent coverage. It should be noted that while this level of accuracy is standard, it still implies a large volume at $z=1$ and therefore our sample selection below likely includes foreground and background interlopers. We have no leverage to remove these without exhaustive spectroscopic data.

\section{SAMPLE SELECTION}

A detailed description of the cluster properties, masses, colormagnitude diagrams, and redshift distributions is given in Paper I (Krick et al. 2008). Table 1 is reproduced here from that paper to summarize their properties. The first cut we make on the sample is that the objects need to have detections in at least six bands to ensure that they are real detections and not noise fluctuations. Because we use ACS locations to measure IRAC fluxes, there are cases where ACS noise (diffraction spikes, etc.) will get picked up as an object with five flux measurements. On the other hand there are real cluster galaxies which are only detected in ACS + IRAC bands because ACS is the deepest band blueward of IRAC and the SEDs are falling sharply into the blue.

We choose twice the virial radius as the interesting physical radius that includes the dense core of the cluster but also the infall region out to roughly the turnaround radius where we might expect to find different populations of galaxies. Cluster centers are determined from the spatial distribution of the member galaxies in the F814W filter. We determine the virial radius from our X-ray detections (see Paper I, Figure 3 for the Chandra image). The diffuse Chandra detections give us $r_{500}$; the radius at which the cluster has 500 times the critical density of the universe. From there we derive $r_{\text {vir }}$ assuming that $r_{500}=0.6 * r_{\text {vir }}$ (Johnston et al. 2007). This relation between $r_{500}$ and $r_{\text {vir }}$ comes from the average of 130,000 groups and clusters from SDSS. For our relatively low-mass clusters $r_{\mathrm{vir}}$ is 0.7 , 
Table 1

Cluster Characteristics

\begin{tabular}{lcccccc}
\hline \hline Cluster & R.A. & Decl. & $N_{\text {gals }}$ & $z_{\text {peak }}{ }^{\text {a }}$ & $\begin{array}{c}L_{x}(0.5-2.0 \mathrm{Kev}) \\
1 \times 10^{43} \mathrm{erg} \mathrm{s}^{-1}\end{array}$ & $\begin{array}{c}M_{500} \\
1 \times 10^{13} M_{\odot}\end{array}$ \\
\hline 1 & J2000 (deg) & J2000 (deg) & $r<500 \mathrm{Kpc}$ & & $2.6 \pm 0.6$ & $6.2 \pm 1.4$ \\
2 & 264.68160 & 69.04481 & 215 & $1.0 \pm 0.1$ & $3.6 \pm 0.7$ & $3.6 \pm 1.4$ \\
3 & 264.89228 & 69.06851 & 255 & $1.0 \pm 0.1$ & $1.6 \pm 0.6 \pm 1.1$ \\
\hline
\end{tabular}

Note.

${ }^{a}$ Redshift peak and $1 \sigma$ uncertainty are measured from a Gaussian fit to the redshift distribution.

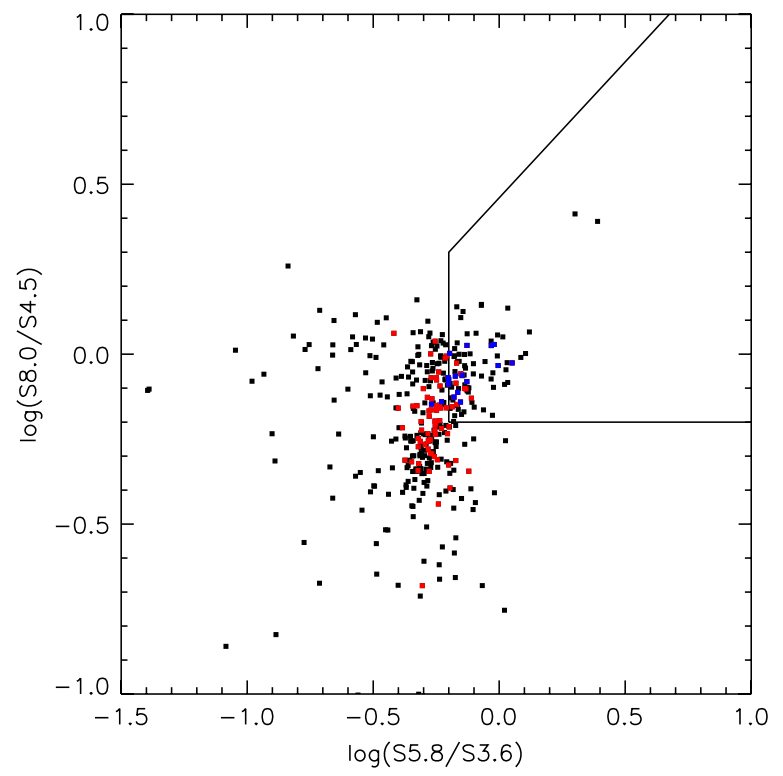

Figure 2. IRAC color-color diagram after Lacy et al. (2004). All cluster member galaxies are shown in black. Those with $24 \mu \mathrm{m}$ detections are color coded red for star-forming and blue for AGN based on HYPERZ fits of their SEDs. Lines show the expected location of AGN based on having red colors in both axes. The member galaxies on the color-color diagram are where we expect redshift one galaxies to be (Sajina et al. 2005) based on position of PAH features and the stellar peak, which is a nice confirmation of our photometric redshifts.

0.58 , and $0.58 \mathrm{Mpc}$, which corresponds to $877^{\prime \prime} .3,72$.' 8 , and $72^{\prime \prime} .8$, respectively. Clusters 2 and 3 are too close to discuss separately as their virial radii are overlapping. We therefore consider them as one structure. The selection area will be the addition of the two circular regions. For cluster 1 we only consider half of the possible total area because the other half is not completely covered by our ACS imaging. While the ACS data are missing, we do have IRAC and MIPS data for this region which indicate that the cluster is symmetric and therefore we are not missing an obvious population by cutting the cluster in half.

Cluster members are chosen by their photometric redshifts. The cluster redshift distributions are centered at $z=1.0$. Our photometric errors at this redshift are 0.13 , so we take as members all galaxies within $0.87<z_{\text {phot }}<1.13$ with HYPERZ chi-squared values less than 50 . This high value cutoff of chisquared is to keep out the catastrophic failures of HYPERz. We do not use the red sequence to determine membership because we expect some of the member galaxies to be blue, particularly those with MIPS detections, and we do not want to bias this work against those galaxies.

Overall there are 443 member galaxies with detections in at least six bands and positions within two virial radii of any of the cluster centers, 90 of those have $24 \mu \mathrm{m}$ detections with $f_{24}>17.3 \mu \mathrm{Jy}$. Because the PSF of MIPS is larger than the IRAC PSF, we checked by eye all MIPS matches for all

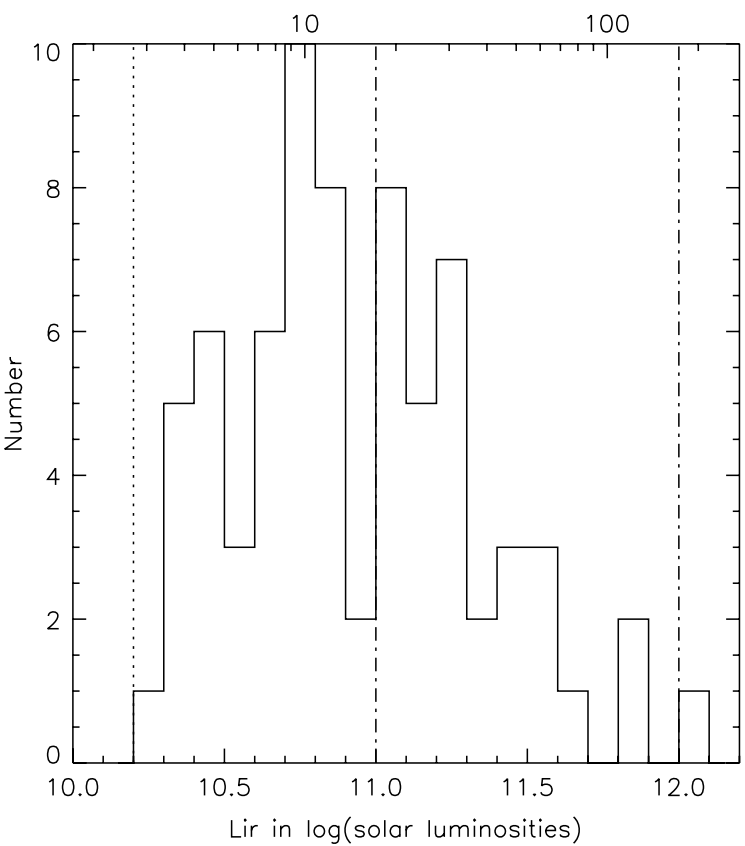

Figure 3. Histogram of IR luminosity of the star-forming member galaxies. The top axis shows SFR in $M_{\odot} \mathrm{yr}^{-1}$. The dot-dashed lines show the cutoff for LIRGs and ULIRGs at $1 \times 10^{11}$ and $1 \times 10^{12} L_{\odot}$, respectively. $43 \%$ of the sample are above the LIRG cutoff. The dotted line shows the completeness limit of the MIPS data.

objects within the area of the clusters to ensure that the correct matches with the closest centers were chosen. In the case of ambiguity, where multiple galaxies could have matched the MIPS source, those sources were not included in the analysis (approximately 10 sources). We also checked by eye those MIPS sources that were not determined to be members to make sure that a mismatch did not occur that would have kept those objects out of the member list. This fraction of members with MIPS detections of $20 \%$ is in the right ballpark when compared to those in the literature given the varying methods of determining membership, varying depths, and different cluster masses. Bai et al. (2007) found that $13 \% \pm 3 \%$ of cluster members are actively forming stars with $f_{24}>50 \mu \mathrm{Jy}$.

Because we have a relatively large area at redshift one in the IRAC dark field, we are also able to make a redshift one "field" sample of those galaxies with the exact same criteria as above (secure detections, $z=1$, and $f_{24}>17.3$ ) except that they are required to be more distant than two viral radii of the cluster centers.

\section{RESULTS AND DISCUSSION}

\subsection{Dominant SED Shape}

Because IR flux can be generated either by dust re-radiating young star light or accretion onto a black hole, we attempt to 
divide the sample into sources where the MIPS flux is likely to be dominated by SF and those where an AGN likely dominates. There is no perfect way to determine this division and it is very likely that sources have signatures of both processes (see Section 1). The best discriminator for the available data is the differing spectral shapes of the UV to mid-IR range for AGNs and galaxies. AGNs have red continua in this range owing to their rising power-law shape as opposed to the falling blackbody in the same wavelength regime for galaxies. We choose to use the SED shapes as fitted by HYPERz to determine if the source spectrum is best fitted by a star-forming galaxy or an AGN.

AGN candidates account for 17 of the 90 member galaxies with $24 \mu \mathrm{m}$ detections or $19 \%$ of MIPS sources and $4 \%$ of all members. These are referred to in the rest of the paper as the AGN sample. The remaining 76 galaxies have SEDs which are dominated by SF and are referred to here as the star-forming member sample. Figure 2 shows the IRAC color-color diagram for all member galaxies as a complementary method of separating AGN from star-forming galaxies (Lacy et al. 2004; Stern et al. 2005). Those galaxies with MIPS $24 \mu \mathrm{m}$ detections are denoted with red (star-forming galaxy) or blue (AGN) colors based on their HYPERZ fits. It is unsurprising to find that the sources tagged as AGNs by their spectral fits also fit into the AGN wedge with $88 \%$ completeness but with significant contamination; $40 \%$. The contamination is likely from intermediate redshift, polycyclic aromatic hydrocarbon (PAH) dominated galaxies and is similar in quantity to simulations by Sajina et al. (2005).

\subsection{AGN Fraction}

We compare here the evolution of the AGN-dominated MIPS sources in clusters with that in the field. These are the first AGN fractions of MIPS-detected sources in clusters at high redshift. MIPS is sensitive to the Compton thick AGNs not detectable at other wavelengths. The literature does hold published Xray-based AGN fractions in clusters. The only other IR work on this topic was published very recently by Galametz et al. (2009) based on observed frame IRAC colors and not mid-IR luminosities. Both the X-ray and near-IR studies find tantalizing evidence for an increasing AGN fraction with increasing redshift (Martini et al. 2007; Eastman et al. 2007; Kocevski et al. 2008; Cappelluti et al. 2005). In a compilation, Eastman et al. (2007) looked at the redshift evolution of the AGN fraction where AGNs were selected from a sample of cluster members with $M_{R}<-20$. X-ray point sources with luminosities above $1 \times 10^{43} \mathrm{erg} \mathrm{s}^{-1}$ were counted in comparison to the member galaxies. They found a trend of AGN fraction increasing from $0.07 \%$ to $2 \%$, an increase of a factor of $\sim 20$, over the redshift range $0.2<z<0.6$. Kocevski et al. (2008) looked at a similar sample of X-ray sources in a supercluster at $z=0.9$ and confirmed the trend of higher AGN fraction at these higher redshifts. Although we have Chandra data which detect diffuse emission from two of the clusters, we are not able to do a point source analysis in the clusters to any meaningful depths.

In order to make a similar AGN fraction measurement to those described above we attempt to make similar flux cuts on our sample to find those galaxies that could potentially host AGNs and the subset of those that we measure to be AGN dominated. We take the potential hosts to be all galaxies at the cluster redshift with $M_{R}<-20$ corresponding to $m_{r}<24.8$, including $\mathrm{K}$ and evolution corrections for early-type galaxies. Although these sources potentially have non-early-type SEDs, we chose those $\mathrm{K}$ and evolutionary corrections to be consistent with what was done in Eastman et al. (2007). Instead of a limit on X-ray luminosity, we use a correlation between $L_{x}$ and $L_{5.8 \mu \mathrm{m}}$ (Fiore et al. 2009) to determine which of our member AGNs have $L_{5.8}>2.5 \times 10^{43} \mathrm{erg} \mathrm{s}^{-1}$. With these requirements we find there are 97 member galaxies within one virial radii of the cluster which have $m_{r}<24.8$. Four of those galaxies have SED shapes of AGNs and $L_{5.8}>2.5 \times 10^{43} \mathrm{erg} \mathrm{s}^{-1}$ or $4 \%$ of the possible hosts. When compared to the Eastman et al. (2007) fraction of $0.07 \%$ at $z=0.2$, our data show an increase by a factor of 60 of the AGN fraction in clusters from redshift 1.0 to 0.2 .

We caution that this fraction depends relatively heavily on the magnitude limit of the sample and the $L_{x}-L_{5.8}$ correlation. If we change the magnitude cut to include fainter (brighter) galaxies down to $m_{r}<25.8\left(m_{r}<23.8\right)$ then we find a ratio of $2.5 \%(6.5 \%)$, both of which still represent an increase over lower redshift clusters but show a large range. If we use the Lutz et al. (2004) relation for the $L_{x}$ and $L_{5.8}$ relation where the $L_{x}>1 \times 10^{43} \mathrm{erg} \mathrm{s}^{-1}$ limit corresponds to $L_{5.8}>3.5 \times 10^{43}$ erg s $\mathrm{s}^{-1}$, then we find a fraction of $1 \%$. We also caution that interpretations about the existence of a trend in the AGN fraction with redshift are limited by the small number the comparison samples. At least for the radio active galaxies, Lin \& Mohr (2007) found that the radio active fraction depends both on the luminosity limit of the sample and the mass of the cluster, such that more luminous galaxies and more massive clusters are likely to have higher fractions of radio active galaxies. They posit that this is really only an effect of the luminosity limit since lower mass clusters are also less likely to have high-luminosity galaxies.

We now compare the evolution of AGN density (number of AGNs per $\mathrm{Mpc}^{3}$ ) in clusters with that in the field. Using the same cluster samples, sample selection, and caveats as above, we calculate that the AGN density in clusters evolves by a factor of $\sim 500$ from $z=1$ to $z=0.2$. This number is also uncertain for the same reasons as mentioned in the previous paragraph and will drop to a factor of $\sim 100$ if the $L_{x}-L_{5.8}$ relation of (Lutz et al. 2004) is used. This measurement of density uses a volume measurement in our data which is a cylinder with depth equal to our redshift uncertainty and a radius of $r_{200}$. We compare this AGN density to a field sample of Ueda et al. (2003) which is a compilation of many surveys with AGNs having $L_{X}>1 \times 10^{43} \mathrm{erg} \mathrm{s}^{-1}$. They found only a factor of $\sim 10$ increase in the field AGN density over the same redshift range. This implies stronger cluster evolution of the AGN density as compared to the field, or that cluster environment has influence over AGN evolution. This same trend is also reported in Eastman et al. (2007) and Galametz et al. (2009). The difference in reported strength is likely due to using different redshift ranges, AGN detection techniques, brightness cutoffs, and the other caveats mentioned above.

\subsection{Star Formation Rate}

Rest-frame $12 \mu \mathrm{m}$ flux correlates with total IR luminosity $\left(L_{\mathrm{IR}}\right)$ which can then be converted into star formation rate (SFR). The correlation between $12 \mu \mathrm{m}$ and $L_{\mathrm{IR}}$ is due to the PAH emission lines. In the absence of longer wavelength data, which is not possible to get at high enough resolution and sufficient depth for these clusters, $12 \mu \mathrm{m}$ is the best wavelength from which to make the conversion; more secure than both 7 and $15 \mu \mathrm{m}$ (Chary \& Elbaz 2001). The correlation between $L_{\mathrm{IR}}$ and SFR comes from the interstellar dust that absorbs the UV-optical light of young stars and re-radiates that energy in the IR. With our $24 \mu \mathrm{m}$ flux and photometric redshifts we 
estimate the total IR luminosity using the methods of Chary \& Elbaz (2001). Specifically templates from both those authors and Dale \& Helou (2002) are redshifted to our source redshift and then matched to the observed $24 \mu \mathrm{m}$ flux. The best-fitting template from each model is then used to derive an average $L_{\mathrm{IR}}$. From there we derive the SFR using the correlation from Kennicutt (1998). The described conversion from $L_{12}$ to SFR is uncertain by factors of a few. However, we note that many of the conclusions of this paper rely not on the absolute SFR, but on the detection of some amount of SF in cluster galaxies.

A histogram of $L_{\mathrm{IR}}$ from the star-forming member sample is shown in Figure $3.43 \%$ of the sample have IR luminosities greater than $1 \times 10^{11} L_{\odot}$ making them luminous infrared galaxies (LIRGs). One galaxy has a flux of $1.01 \times 10^{12} L_{\odot}$ qualifying it to be an ultraluminous infrared galaxy (ULIRG). We find a similar ratio of LIRGs to sub-LIRGs as other clusters at higher redshift. Marcillac et al. (2007) found 60\% of their star-forming sample (30 galaxies) at $z=0.83$ are LIRGs to a very similar detection limit. In a different cluster at $z=0.83$, Bai et al. (2007) found $41 \%$ of their sample (34 galaxies) are LIRGs. However, that survey is not as deep which means there will be more sub-LIRGs which will make this fraction lower. Geach et al. (2006) in two clusters at $z \sim 0.4 \& 05$ do not go deep enough to get a good sample of sub-LIRGs.

We compare the luminosity distributions of star-forming cluster members to field MIPS-detected galaxies at redshift one. A K-S test between the two distributions shows them to have a $99 \%$ probability of being drawn from the same population. This would imply that the cluster environment does not affect the IR luminosity of the galaxies within it. In other words, among star-forming galaxies, SF does not vary with environment.

In addition to calculating individual SFRs per galaxy we compare the total SFR per cluster with other clusters at varying redshifts from the literature. The interesting physical quantity to compare is the mass-normalized SFR because SFR could vary with mass of the cluster (although see Goto 2005). We compare our redshift one clusters with 14 clusters with $0.02<z<0.83$ from the literature (Bai et al. 2007, and references therein). The literature sample selects only those galaxies with SFR > $2 M_{\odot} \mathrm{yr}^{-1}$ within $0.5 r_{200}$. Our SFR cutoff is similar $\left(3 M_{\odot} \mathrm{yr}^{-1}\right)$ and we truncate our sample to match the $0.5 r_{200}$ radius.

In Figure 4 the literature clusters are shown with triangles and the composite of our redshift one clusters with an asterisk. Error bars in all cases are $1 \sigma$ errors taken from the combination of both mass and SFR errors. Our three clusters are relatively low-mass clusters, and because there is some concern about a relation between mass-normalized SFR and mass, we also denote the other lower mass clusters $\left(M<5 \times 10^{14} M_{\odot}\right)$ in this figure with squares. These lower mass clusters in the comparison sample are still of higher mass than our redshift one clusters. However, hierarchical formation tells us that redshift one clusters will grow in mass by the time they reach redshift zero. Comparing clusters of the same mass across a large redshift range would then also introduce a bias into the sample. Our three redshift one clusters are suggestive of continuing the trend of higher redshift clusters having a larger amount of mass-normalized SFR. This is true both when looking at the whole sample of lower redshift clusters and also confining the sample to the five lowest mass, lower redshift clusters. It will be important to compare our clusters to even lower mass, low-redshift counterparts when that data become available.

SFR can also be computed from different wave bands. A detailed discussion of the varying methods and their relative

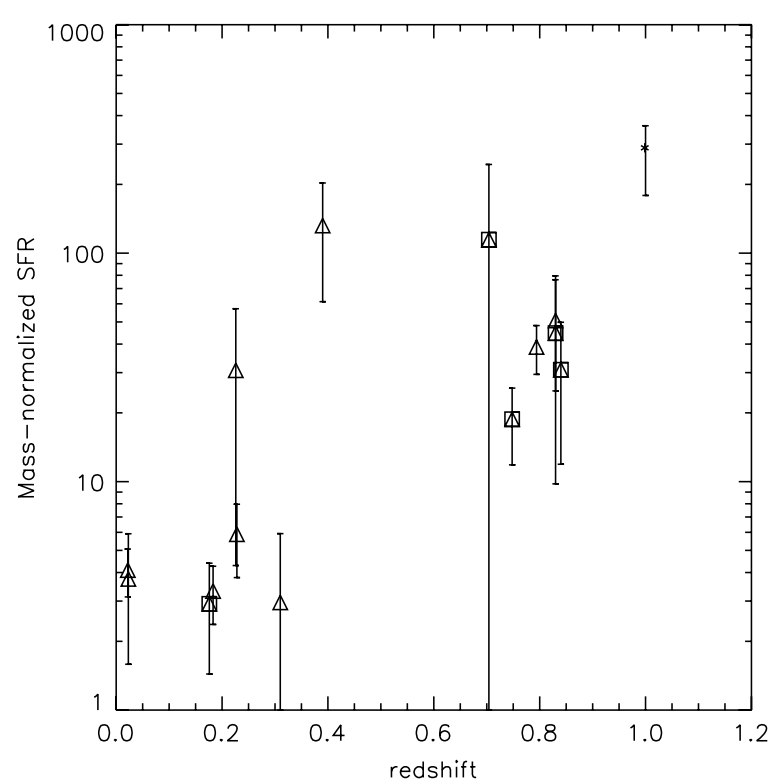

Figure 4. Mass-normalized SFR as a function of redshift. The asterisk represents the three redshift 1 clusters from this survey. The triangles are from the literature. Those literature clusters with masses less than $5 \times 10^{14}$ have their triangles surrounded by squares. Error bars come from a combination of mass and SFR errors.

strengths and weaknesses is beyond the scope of this paper, but see Kennicutt (1998) and references thereto for such a discussion. We would like to compare our results on the redshift evolution of the mass-normalized SFR with other measures from the literature, however such measures are not published covering the entire redshift range presented here. $\mathrm{H} \alpha$ and $\mathrm{O}$ II derived SFRs for clusters at $z<0.8$ are presented in Finn et al. (2008) and Poggianti et al. (2008) and those are in agreement with the mid-IR determined values (Bai et al. 2007).

The comparison with the field SFR evolution is also interesting. We know that the SFR density (SFRD) in the universe peaks around $1<z<2$ and then declines to today (Madau et al. 1998; Lilly et al. 1996). In a recent compilation of SFRD measurements, Hopkins \& Beacom (2006) show a factor of $5 \sim 10$ drop in the SFRD from redshift 1.0 to 0.1 in the field. We calculate the SFRD of our clusters at $z=1$ and compare this to the SFRDs for the four lowest redshift clusters in the literature sample with an average redshift of $z=0.1$. We do this using the sample confined to $0.5 r_{200}$ for ease of comparison. We find a drop in SFRD from $z=1.0$ to $z=0.1$ of a factor of 40 . This could imply that while the distribution of IR luminosities of $z=1$ cluster galaxies is similar to the field, the suppression of SF happens more quickly in clusters than in the field, implying that the cluster environment is more efficient in the suppression of SF and AGNs than the field. Our data suggest this is the case, but a larger, more uniform sample is required for confirmation.

\subsection{Color}

We explore the colors of the MIPS-detected, star-forming sources in the clusters for the purpose of understanding if the red galaxies in the clusters are red because they have no SF, or if they are red due to dust. Figure 5 shows the distribution of rest-frame $B-K$ colors of the MIPS-detected, star-forming member galaxies (dashed line) and all cluster members (solid line). We use the dotted line as the dividing line between the blue cloud and the red sequence (see Paper I). 

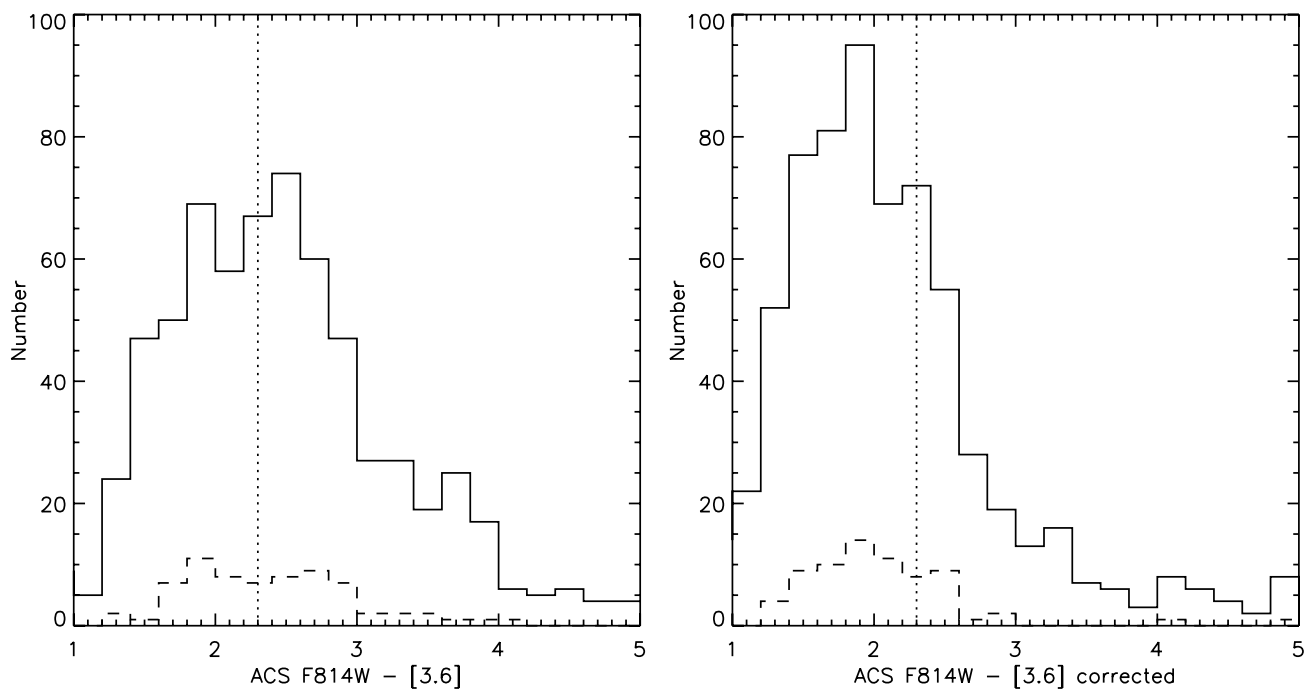

Figure 5. Histogram of colors of the star-forming member galaxies (dashed) and all member galaxies (solid). At redshift one this color range is rest-frame $B-K$. The vertical dotted line shows roughly where the division between red and blue galaxies lies. The right figure shows the color distribution of the same samples where colors are corrected for extinction based on SED fitting and a Calzetti extinction law.

We correct galaxy colors for dust reddening using the extinction as measured by the HYPERZ SED fits and the Calzetti et al. (2000) extinction law. Another possible way to make this correction is with Balmer line spectroscopy. However, with a sample of greater than 2000 galaxies at $0.05<z<1.5$, Cowie \& Barger (2008) found that the SED fitting is a comparable technique and in fact used the SED fitted extinction instead of the Balmer ratios even when they do have spectroscopy. The corrected colors for our sample are shown on the right side of Figure 5.

There is a significant amount of extinction at these wavelengths, particularly at rest-frame $\mathrm{B}$ where extinctions range from $A_{B}=0-1.6$, showing that many of these galaxies are dust reddened star-forming sources and in large part not galaxies that are red due to age. The corrected histogram shows a very different distribution, with $57 \%$ of the MIPS sources moving from the red sequence to the blue cloud. This is consistent with Cowie \& Barger (2008) who found roughly half of their MIPSdetected red-sequence galaxies move off of the red sequence after correction. These data tell us that the MIPS sources do not form a uniquely colored population and are instead very dusty galaxies.

\subsection{Morphology}

We examine morphologies of the MIPS sample both with an SED fitting and a by-eye determination for the purpose of determining which types of galaxies are mid-IR bright in clusters at $z=1$. One method of determining galaxy type is by fitting templates to its SED. This really is a measure of the SED shape used as a proxy for morphology. The strength of this method is that it allows us to easily compare the field to cluster galaxies using the same objective criteria. The SED shape has already been determined for all galaxies with HYPERZ while fitting for photometric redshifts. In Figure 6 we show the histogram of types of galaxies from this analysis arranged from star-forming galaxies to AGNs. The solid line shows the member galaxies with $24 \mu \mathrm{m}$ detections and the dashed histogram is the normalized histogram of all $24 \mu \mathrm{m}$ detections across the entire field. As expected, there are relatively few early-type galaxies, and a relatively large number of late-types and AGNs.

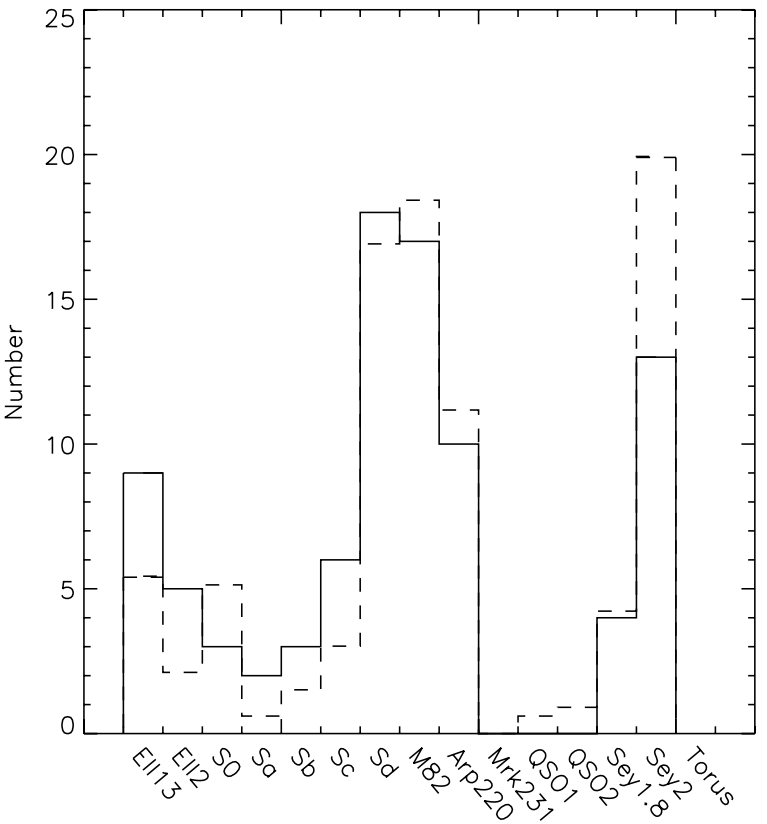

Figure 6. Best-fit morphologies from HYPERZ SED fits. The solid line shows the member galaxies with $24 \mu \mathrm{m}$ detections and the dashed histogram is the normalized histogram of all field $24 \mu \mathrm{m}$ detections.

There are very similar distributions from cluster to field. This is perhaps hinting that the cluster environment is not effecting the morphologies of the mid-IR bright galaxies, much like the IR luminosities of star-forming galaxies being unaffected by environment in Section 5.3.

Since there are only 90 galaxies with MIPS detections at the cluster redshift, we classify their morphologies manually by eye. For this, we use the data with the best resolution which are the HST ACS F814W data, corresponding to restframe $B$ band, with $0^{\prime \prime} .5$ pixel $^{-1}$ resolution. Training for this was done with examples from the online SDSS GalaxyZoo ${ }^{6}$ which has color images at a range of redshifts. We choose a very simple classification scheme meant to divide those galaxies

\footnotetext{
6 http://www.galaxyzoo.org/
} 


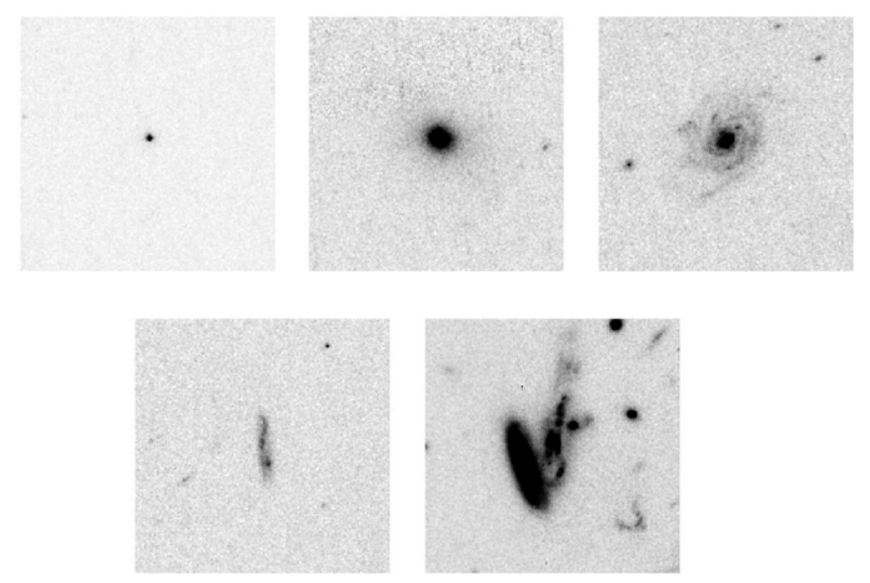

Figure 7. Examples from HST ACS F814W of each type in our morphological classification: compact, elliptical, spiral, irregular/disk, and irregular/merger All thumbnails are $10^{\prime \prime}$ on a side. The irregular/merger example comes from the center of cluster 2 and is our only ULIRG.

with visible signs of interactions from those without. To this end we choose five categories which fit all galaxies with the exception of seven galaxies because they were either not imaged with ACS or are too near a bright star or its diffraction spikes to clearly classify. The five categories are Compact, Elliptical, Spiral, Irregular/disk, and Irregular/merger. We stick to very basic definitions to avoid ambiguous classifications. Things fall into the compact, elliptical, or spiral classes if they have classical forms of those shapes. Although compact classified objects have the shape of a PSF, they have been confirmed to be nonstellar based on their SED fits. Spirals include anything with a disk that does not look disturbed or asymmetric in any way. Irregular galaxies are anything that does not fit one of the classical descriptions. Because the Irregular galaxies make up such a large fraction of the sample, we have subdivided that classification into those systems that clearly have multiple nuclei or obvious tidal tails (Irregular/merger) and all other irregular galaxies, mainly disturbed disks (Irregular/disk). This differentiation of the irregular galaxies may indicate something about the timescales of interaction histories with the Irregular/ merger classification going to those objects at earlier stages of interaction, and Irregular/disk going to those objects at later stages. Figure 7 shows examples from our sample of our morphological classification.

Table 2 shows the morphological distribution for the entire sample as well as subsamples based on color and IR luminosity (Sections 5.4 and 5.3). Of the entire sample of member galaxies with MIPS detections, the majority of them are either spirals or irregulars $(81 \%)$, unsurprisingly. Specifically, $25 \%$ of the sources show obvious signs of interactions or mergers. There are potentially more interacting galaxies whose tidal features are too low in surface brightness for us to detect but this cannot account for all of them. In the cases of the galaxies which show signs of interaction we do not need to invoke a cluster environment driven process to trigger SF, we can assume here that the merger has triggered SF. The remainder $(75 \%)$ of the sources which do not show signs of interaction must have had their SF triggered by some physical process that can occur within the cluster environment such as ram pressure stripping or harassment (Gunn \& Gott 1972; Moore et al. 1996). In the next section, we discuss the location of this SF to determine if it is on the cluster outskirts and therefore is potentially residual SF after suppression upon entering the cluster environment, or if it is truly being triggered by some cluster process ongoing
Table 2

Morphologies of MIPS24 Members

\begin{tabular}{lccc}
\hline \hline Galaxy Morphology & All & LIRGs & Sub-LIRGs \\
\hline Compact & $\mathbf{9 ( 1 1 \% )}$ & $2(5 \%)$ & $7(16 \%)$ \\
Elliptical & $\mathbf{7 ( 8 \% )}$ & $2(5 \%)$ & $5(11 \%)$ \\
Spiral & $\mathbf{3 0 ( 3 5 \% )}$ & $18(44 \%)$ & $12(27 \%)$ \\
Irr/Disk & $\mathbf{1 8 ( 2 1 \% )}$ & $9(22 \%)$ & $9(21 \%)$ \\
Irr/Merger & $\mathbf{2 1 ( 2 5 \% )}$ & $10(24 \%)$ & $11(25 \%)$ \\
Total & $\mathbf{8 5 ( 1 0 0 \% )}$ & $41(100 \%)$ & $44(100 \%)$
\end{tabular}

Notes. The first data column shows the morphology breakdown for all member galaxies with MIPS detections. Columns 2 and 3 divide all members into those with LIRG and sub-LIRG luminosities. Percentages are of the galaxies only within the column shown.

inside the cluster and suppression is not complete at the cluster edges.

The majority of compact sources are part of the AGN sample based on SED fitting. Other compact determined galaxies are likely ellipticals where the lower surface brightness outer parts of the bulge are not visible at $z \sim 1$.

The ellipticals are an interesting population in which to find SF. From our original sample selection of 443 member galaxies, less than $2 \%$ are ellipticals with MIPS detections. About half of the elliptical galaxies have red colors both before and after extinction correction implying that there are a few legitimate red ellipticals with SF signatures. Some of these are possibly misclassifications because of projections or surface brightness dimming of a disk component or AGN misclassifications. Most of these ellipticals are sub-LIRGs so they do not have the higher SFRs in the sample. It is possible that we are seeing residual SF after a merger, but it is hard to imagine that the morphological change would precede the end of the triggered SF. The last possibility is that we see signs of dusty SF in elliptical galaxies that go against traditional findings that elliptical galaxies have no $\mathrm{SF}$, at least not at the SFRs to which we are sensitive $\left(>3 M_{\odot}\right)$. Optically red, morphologically elliptical galaxies with excess $24 \mu \mathrm{m}$ emission have also been found in SWIRE, GOODS, and the Bootes fields (Rodighiero et al. 2007; Davoodi et al. 2006; Brand et al. 2009). While some of these show AGN signatures, some are attributed to SF.

When we split the sample based on IR luminosity we see that the spirals and irregulars make up the majority of the LIRGs (90\%) but a lesser percentage of the sub-LIRGS $(73 \%)$ due to the higher fraction of compact and elliptical sources. Also interesting is that the irregular population is split evenly between LIRGs and sub-LIRGs, and $60 \%$ of the spirals are LIRGs. In summary, LIRGs in clusters are most likely to be blue spirals or irregulars. Dividing by morphology, spirals are more likely to be LIRGs, irregulars are equally likely to be LIRGs or sub-LIRGs, and ellipticals are most likely sub-LIRGs.

Our findings of the ratios of morphological types in clusters is similar to other published cluster values at high redshifts. In their survey of a redshift 0.83 cluster, Bai et al. (2007) find that of their IR-detected galaxies, $20 \%, 63 \%$, and $16 \%$ of them are early-type, late-type, and irregular galaxies, respectively, and $32 \%$ show signs of mergers/interactions. Also for a redshift 0.83 cluster, Marcillac et al. (2007) found $75 \%$ spirals (including S0s, since we would have given those a spiral designation) and $25 \%$ irregulars. Again these are only rough comparisons with the caveat that all of these studies have only small samples which vary in cluster mass, density, and dynamical state, etc., all things which might have an effect on the morphologies and IR luminosities of member galaxies. 

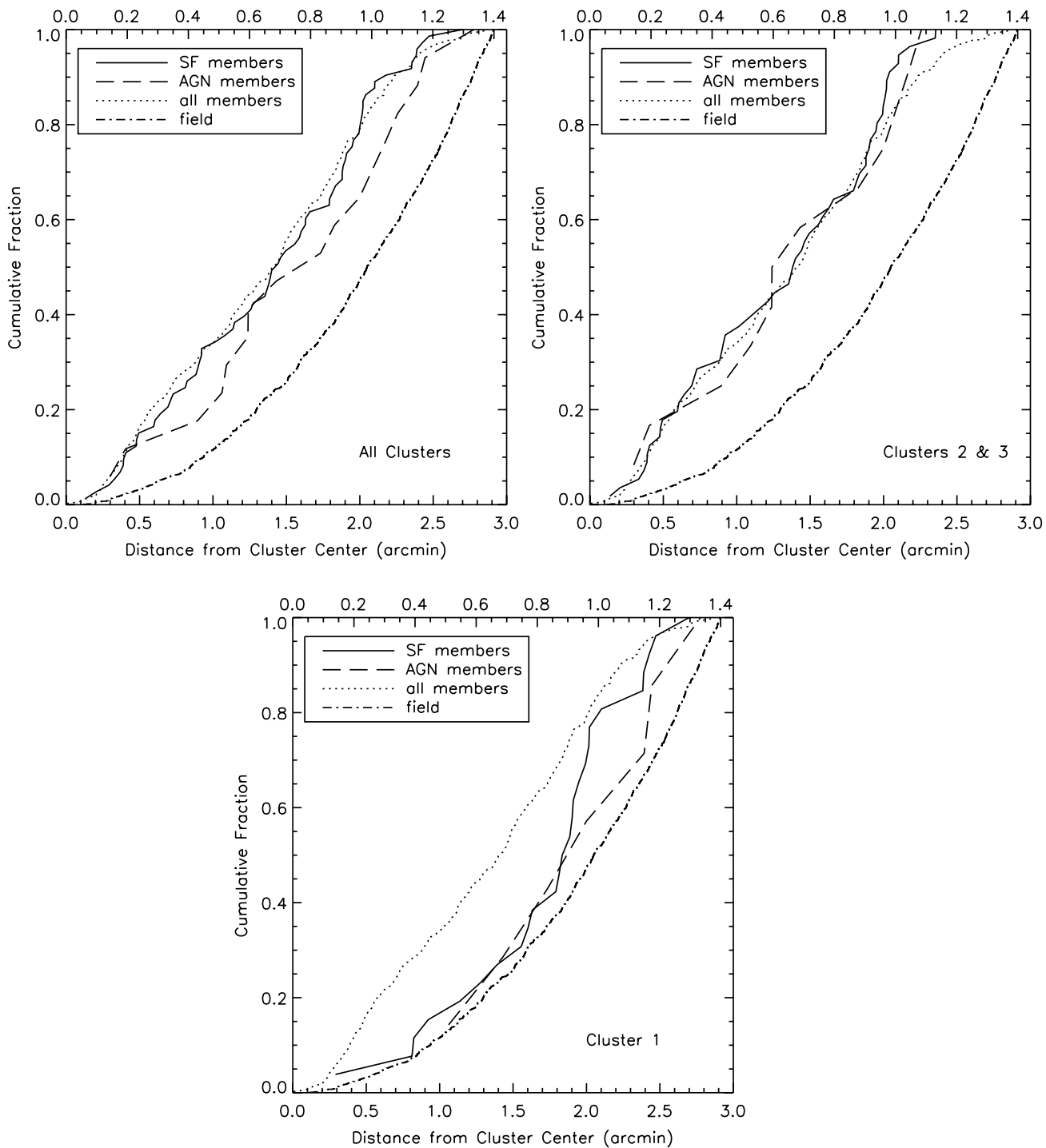

Figure 8. Cumulative distribution functions with distance from cluster center reported in arcminutes on the bottom axis and Mpc on the top axis. The solid, dashed, dotted, and dot-dashed lines represent the cluster star-forming members, the cluster AGN members, all cluster members without MIPS flux or AGN SED shapes, and a field sample at redshift one, respectively. Top left: all three clusters combined; top right: clusters 2 and 3 only; bottom: cluster 1 only.

\subsection{Distribution of Star Forming Galaxies}

We examine the location of the MIPS sources in the clusters with the goal of measuring if they are more or less concentrated than the non-MIPS sources which would imply that they preferentially live in the centers or outskirts of the clusters. We make this comparison using cumulative distributions and a $\mathrm{K}-\mathrm{S}$ test which is the most straightforward way to determine if two continuous, unbinned, distributions are drawn from the same parent distribution. This is the best statistical test to make this measurement given a relatively small sample of galaxies especially when we split the sample by galaxy property to examine the trends below. $\mathrm{K}-\mathrm{S}$ tests are relevant on samples sizes larger than $\sim 5$ (Press et al. 2007). Figure 8 shows unbinned cumulative distributions as a function of the distance from the cluster center. All three clusters are combined here on the top left panel and the distance from the center is taken to be distance to the nearest cluster center. In the top left panel we show the distribution of the MIPS-detected star-forming galaxies (solid) and AGN (dashed) compared to both all cluster members (dotted) and the field (dot-dashed). We check that increasing the sample to include objects with "only" five flux detections does not change the shape of the cumulative distribution (see Section 4 for a discussion of the number of detections required for an object to be included in the sample).

The first thing to note is that all the cluster samples (those with and without MIPS detections) show evidence of being significantly more centrally concentrated than a comparison field sample as measured in circles of the same area in the field. A K-S test on the composite sample shows less than 1E-7\% chance that they are drawn from the same parent population. This is both nice confirmation of our photometric redshifts and proof that SF occurs in cluster environments. In a similar experiment we determine the space density of MIPS sources in the composite tri-cluster area compared to a similar area in the field. In field regions of the same area as the cluster, we measure the mean space density to be $43 \pm 15$ sources whereas we detect 90 sources in the cluster area which is a greater than $3 \sigma$ overdensity. The cluster environment has clearly enhanced the number of mid-IR sources among its member galaxies. This is usually, but not always the case in the literature. Geach et al. (2006) found only a very minimal overdensity in MS0451-03 
at $z=0.55$. Marcillac et al. (2007); Bai et al. (2007); Gallazzi et al. (2009) all find significant overdensities when compared to the field.

Second, the top left panel of Figure 8 shows that the starforming MIPS members and the non-MIPS-detected members are consistent with having the same spatial distribution. A K-S test shows they have a $97 \%$ chance of being drawn from the same population. We investigate this trend further by dividing our cluster sample. The top right and bottom panels of Figure 8 show the cumulative distributions for the separated clusters. Interestingly, cluster 1 on its own has a significantly different spatial distribution which has only a $1 \%$ probability of being drawn from the same population as the rest of the member galaxies. In this cluster we see a less concentrated distribution of star-forming galaxies until roughly 1 virial radius $(0.7 \mathrm{Mpc})$ at which point the distribution steeply rises, indicating a possible excess of star-forming galaxies just beyond that radius.

The other two clusters show no such trend. One possible explanation is that there is some critical cluster property different between these two sets driving the difference in spatial distributions. One could imagine that cluster property to be mass or evolutionary state. Cluster 1 is both more massive than the other clusters and is more relaxed in the sense that it appears to have already formed a $\mathrm{cD}$ galaxy whereas the other clusters are in the process of forming their cDs (see Figure 7 for an image of the central galaxy in cluster 2). A larger sample is required to examine these differences. A second possibility is that these two clusters represent a complex structure in our two-dimensional image. They are relatively close to each other (overlapping virial radii at the same photometric redshifts) that it is possible these two clusters actually reside in the same potential well, or that one is falling in toward the center of the other, which would make our choice of centers meaningless. Because of their nearness, we could imagine that projection effects could dilute any potential signal of a less concentrated distribution.

We have found that different clusters potentially exhibit different spatial distributions in their star-forming galaxies, which is also found in Geach et al. (2006). Coia et al. (2005), Bai et al. (2007), Marcillac et al. (2007), Gallazzi et al. (2009), Fadda et al. (2008), and Koyama et al. (2008) reported the detection of an intermediate density at which cluster star-forming galaxies congregate, but this is also not found in the work of Biviano et al. (2004). The comparison of literature samples is not straightforward because of the differing cluster properties (mass, virialization, and structure) and differing sampling methods including flux detection levels and accounting for AGN contamination. Also in some cases the evidence for star-forming galaxies to preferentially lie at intermediate densities is not statistically strong $(<3 \sigma)$. For these listed reasons, and that different authors use different measures of local environment, it is not practical to compare literature samples.

We further discuss the cumulative distributions of the sample with a focus on IR luminosity, morphology, and color. We continue to discuss the sample as the combination of all three clusters which does not effect the remainder of the results.

\subsubsection{Distribution by $L_{I R}$}

We divide the sample of star-forming galaxies based on IR luminosity in the top right panel of Figure 9. Those with LIRG luminosities or above are shown with the solid line, and those with sub-LIRG luminosities are shown with the dotted line. The LIRGs do appear to be more centrally concentrated than the sub-LIRGS, however a $\mathrm{K}-\mathrm{S}$ test is inconclusive giving a $53 \%$ probability that they are drawn from the same population. This inconclusiveness means that we cannot rule out the possibility that sub-LIRGs have a different, less concentrated, distribution than LIRGs. This leaves open the possibility that at lower redshift where surveys are likely to be deeper than high-redshift surveys, the lower luminosity sub-LIRGS might dominate the population thereby giving the appearance of being overall less concentrated than the other member galaxies. This could be a reason why lower redshift surveys find less concentrated spatial distributions, but does not explain the preferred density peaks reported in those studies.

\subsubsection{Distribution by Morphologies}

We now investigate the location of the star-forming galaxies by splitting the sample on morphology. If the spirals are less centrally concentrated it could suggest that the cluster environment is able to burst and then suppress SF in normal noninteracting galaxies. The remaining SF activity that we see closer to the center is then the result of galaxy interactions. The top right panel of Figure 9 shows the cumulative distribution of the spiral sample (solid line) and the likely merger sample (dotted line), based on the morphologies as determined by eye in Section 5.5. There is again tantalizing but inconclusive evidence that the spirals are less centrally concentrated. A K-S test on these two samples gives a $65 \%$ chance that the two populations draw from the same parent distribution which prevents us from concluding either that they are or are not more centrally concentrated. We also plot the distribution of the elliptical starforming galaxies but small sample size (seven galaxies) prevents us from making conclusions. Projection effects also complicate this analysis since we do not know the three-dimensional location of the member galaxies.

The inconclusive tests for both morphology and $L_{\mathrm{IR}}$ radial distributions are probably telling us that there is another variable which is confusing these tests. A larger sample size of clusters split by cluster properties is desirable to further test our hypotheses in these cases.

\subsubsection{Distribution by Color}

To understand more about spatial distribution of the MIPS sources we further divide the sample of star-forming galaxies by color into a red and blue sample based on their uncorrected magnitudes. The dividing line is taken to be the blue edge of the red cluster sequence as described in Paper I and shown in Figure 5. The same division is made for the non-MIPS-detected cluster members and the results are shown in the bottom left panel of Figure 9. The left, more centrally concentrated fork of the distribution shows the red galaxies while the right fork shows the distribution of the blue galaxies with solid lines for the MIPS members and doted lines for the non-MIPS member galaxies. Again we see that the MIPS members and non-MIPS members show similar distributions $(\mathrm{K}-\mathrm{S}=76 \%$ for red and $70 \%$ for blue) while we see a clear difference between red and blue galaxies $(\mathrm{K}-\mathrm{S}<0.01 \%)$ with blue avoiding the central dense regions of the clusters. This is a classic finding that blue galaxies generally do not inhabit dense environments (Butcher \& Oemler 1984; Pimbblet 2003).

However, if we look at the distribution of the reddening corrected colors (Section 5.4) we find a different story; bottom right panel of Figure 9. Here we see that the blue non-MIPSdetected members still show the same trend of the blue galaxies avoiding the centers. In contrast to the nonreddening-corrected color distributions, all the MIPS-detected galaxies now have the 

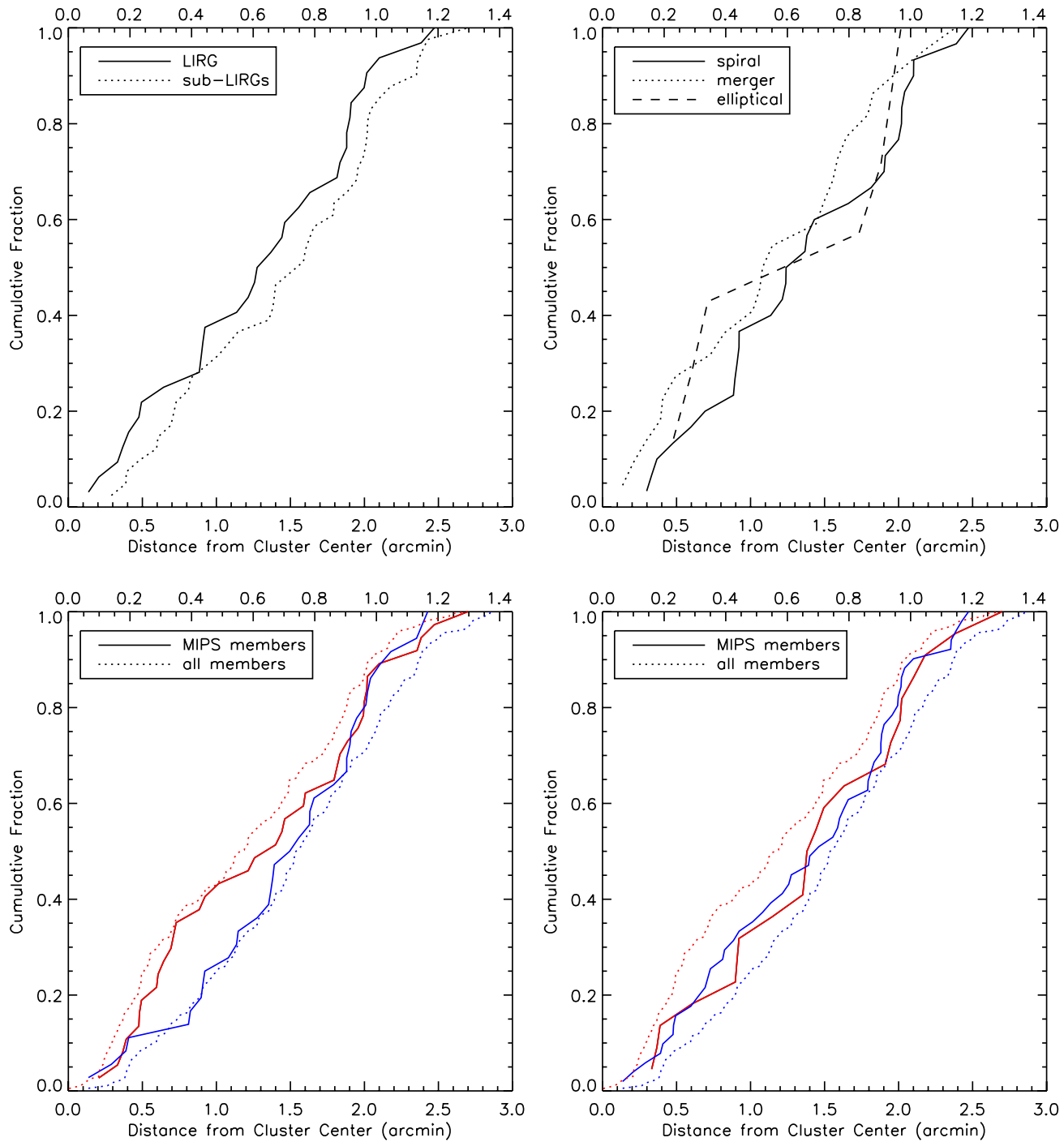

Figure 9. Cumulative distribution functions with distance from cluster center reported in arcminutes on the bottom axis and Mpc on the top axis. Top left: distribution split by IR luminosity. LIRGS (including the lone ULIRG) are shown with the solid line, while sub-LIRGS are shown with the dotted line. Top right: distribution split by morphology into spirals (solid), ellipticals (dashed), and irregular/mergers (dotted). Bottom left: distribution split by color, uncorrected for reddening. The solid and dotted lines represent the cluster star-forming members and all cluster members without MIPS flux or AGN SED shapes. The more concentrated set of solid and dotted (red) lines represent the red galaxies while the less concentrated (blue) set of lines show the blue galaxies. Bottom right: distribution split by reddening-corrected color; same line definitions as the middle right plot.

same distribution regardless of color $(\mathrm{K}-\mathrm{S}=94 \%)$. In other words, the formerly red galaxies are co-spatial with the blue galaxies. This is just showing us again that many of the observed red galaxies are actually dusty blue galaxies and are not red because they are old.

\subsection{Distribution of AGN}

We examine the distribution of cluster AGNs compared to the MIPS-detected star-forming galaxies. Figure 8 includes the cumulative distribution of MIPS-detected AGN cluster members as the dashed lines. A K-S test between the AGNs and star-forming members in the combined distribution is inconclusive, showing a 50\% probability of drawing from the same population. A K-S test on clusters 2 and 3 shows a $90 \%$ probability of deriving from the same population. It is interesting that the AGN and star-forming galaxies appear to have similar distributions in two of the clusters. The similar distribution could imply that the same physical mechanism triggers AGNs and SF. In a similar case to the SF galaxies, because cluster 1 shows a different AGN distribution from the other clusters, we are unable to ferret out the underlying causes of the distributions. The literature is similarly inconclusive. A radio sample from Lin \& Mohr (2007) shows that AGNs are more concentrated than cluster galaxies with the radio-brightest being the most concentrated. A sample of eight low-intermediate redshift clusters with X-ray-detected AGNs reveals the same trend (Martini et al. 2007). However, in a supercluster at $z=0.9$, Kocevski et al. (2008) found that X-ray AGNs are more likely located in the intermediate regions, avoiding the densest cluster centers. The differences in samples between Radio, X-ray, and mid-IR selections and differences between depths and cluster characteristics may be the source of these differences. A larger sample is necessary to make progress on this topic.

\section{CONCLUSION}

We have used a multiwavelength data set based on extremely deep Spitzer IRAC data to examine the nature of mid-IR sources in a large-scale structure of three clusters at redshift 1 . There 
are 90 members of the clusters with MIPS detections within two virial radii of the cluster centers, of which 17 appear to have SEDs dominated by AGNs and the rest dominated by SF. With the samples of AGN and star-forming sources we examine the total IR luminosities, SFRs of individual galaxies and of the structure as a whole, colors, morphologies, and distributions whereby we come to the conclusions listed below.

1. We look for evolution in the AGN fraction with redshift. In a comparison with X-ray surveys we find a continued increase in the AGN fraction out to redshift 1 with trepidation over the accuracy of the conversion between $L_{5.8}$ and $L_{X}$. In addition the magnitude of the increase in the AGN fraction is higher in clusters than in the field. If an effect of AGN activity is to suppress SF through a feedback mechanism, then the measured large number of AGNs at higher redshifts indicates that there will be many galaxies for which AGN feedback may be a significant player in turning off SF in lower redshift clusters. Second, because of the more rapid decrease in the AGN fraction in clusters compared to the field, we conclude that the cluster environment has an effect on the decline of the AGN population.

2. For the sample of star-forming members, we use the $24 \mu \mathrm{m}$ flux (rest frame $12 \mu \mathrm{m}$ ) to estimate total IR luminosity. The distribution of IR luminosities shows that about half of the sample have IR luminosities consistent with being LIRGs while the other half are sub-LIRGs. That distribution is consistent with the field at redshift 1 , as measured from other regions in our data, implying that the cluster environment does not have an effect on the IR luminosities of the galaxies within it.

3. Total IR luminosity is converted to SFR. As a whole, the summed, mass-normalized cluster SFR is higher at $z=1$ than in counterparts at lower redshift. The measured decrease of SFRD from $z=1$ to 0 is larger than the decrease measured in the field implying that suppression of SF is accelerated in the cluster environment.

4. Based on SED-fitted extinction values at rest-frame $B$ band, we find that MIPS sources in clusters are mainly highly extincted, dusty, intrinsically blue galaxies. A few are intrinsically old red galaxies.

5. Morphologies of the MIPS-detected sources are determined by eye from the $H S T$ rest-frame $B$-band images. The majority of sources $(81 \%)$ are spirals or irregulars. There are a few elliptical galaxies $(8 \%)$, the majority of which have sub-LIRG luminosities. Potentially some of these are misclassifications, but some are real detections of dusty SF of greater than three solar masses per year in an elliptical galaxy. The LIRGs in clusters are most likely to be blue spirals or irregulars. A large fraction (at least 25\%) show obvious signs of interactions. This implies that some cluster galaxies have SF triggered by the cluster environment and not solely by merger processes which are not cluster environment dependent.

6. Cluster MIPS sources are significantly more concentrated than a field sample at redshift 1 showing that they are indeed members of the cluster. Cluster characteristics appear to influence the spatial distribution of the starforming member galaxies. One of our clusters shows the MIPS sources with a less concentrated distribution than the other members. However, the other two clusters have MIPS sources with the same distribution as the member galaxies implying that complete suppression has not occurred due to the cluster environment. There is inconclusive evidence for
LIRGs and irregular galaxies separately to be more centrally concentrated than sub-LIRGs and spirals, respectively. When using uncorrected magnitudes, galaxies blueward of the red sequence are significantly less concentrated than red galaxies. However, when using reddening-corrected galaxy colors, we find all MIPS-detected cluster members to have the same distribution confirming that the MIPS sources really are dusty, star-forming, blue galaxies and not a separate population.

Cluster environment does seem to have an effect on the evolution of AGN fraction and SFR from redshift 1 to the present, but among the IR active galaxy sample, environment does not affect the IR luminosities. This may be saying that whatever triggers the SF in clusters has the same effect on the galaxies in the clusters as whatever triggers SF in the field, e.g., SF looks the same regardless of environment. Or, in other words, the effect of SF on a galaxies IR luminosity is independent of triggering mechanism. But, the cluster environment does encourage SFR and the AGN fraction to decline more rapidly with time over the field implying that the cluster environment does have an effect on the activeness of galaxies, either SF or AGN. While some of our galaxies show signs of interaction as the likely triggering mechanism, it seems likely that other cluster environment driven effects are also able to trigger SF within the cluster. This is based both on morphological indicators of SF and the distributions of SF galaxies. In two clusters we see no evidence for a suppression of SF in the inner regions of the clusters as we would expect if there were a density cutoff for triggering SF. As always, a larger sample of clusters with deeper mid-IR measurements is desirable.

This research has made use of data from the Two Micron All Sky Survey, which is a joint project of the University of Massachusetts and the Infrared Processing and Analysis Center/California Institute of Technology, funded by the National Aeronautics and Space Administration and the National Science Foundation. This work was based on observations obtained with the Hale Telescope, Palomar Observatory as part of a continuing collaboration between the California Institute of Technology, NASA/JPL, and Cornell University, the Spitzer Space Telescope, which is operated by the Jet Propulsion Laboratory, California Institute of Technology under a contract with NASA, the MMT Observatory, a joint facility of the Smithsonian Institution and the University of Arizona, and the NASA/ ESA Hubble Space Telescope, obtained at the Space Telescope Science Institute, which is operated by the Association of Universities for Research in Astronomy, Inc., under NASA contract NAS 5-26555. These observations are associated with program \#10521. Support for program \#10521 was provided by NASA through a grant from the Space Telescope Science Institute, which is operated by the Association of Universities for Research in Astronomy, Inc., under NASA contract NAS 5-26555.

Facilities: Hale (LFC, WIRC, COSMIC), MMT (Megacam), HST (ACS), Spitzer (IRAC, MIPS), Akari, CXO (ACIS)

\section{REFERENCES}

Adami, C., Ilbert, O., Pelló, R., Cuillandre, J. C., Durret, F., Mazure, A., Picat, J. P., \& Ulmer, M. P. 2008, A\&A, 491, 681

Bai, L., et al. 2007, ApJ, 664, 181

Bekki, K., \& Couch, W. J. 2003, ApJ, 596, L13

Bertin, E., \& Arnouts, S. 1996, A\&AS, 117, 393

Biviano, A., et al. 2004, A\&A, 425, 33

Bolzonella, M., Miralles, J.-M., \& Pelló, R. 2000, A\&A, 363, 476 
Brand, K., et al. 2009, ApJ, 693, 340

Brodwin, M., et al. 2006, ApJ, 651, 791

Butcher, H., \& Oemler, A. 1984, ApJ, 285, 426

Calzetti, D., Armus, L., Bohlin, R. C., Kinney, A. L., Koornneef, J., \& StorchiBergmann, T. 2000, ApJ, 533, 682

Calzetti, D., Kinney, A. L., \& Storchi-Bergmann, T. 1994, ApJ, 429, 582

Cappelluti, N., Cappi, M., Dadina, M., Malaguti, G., Branchesi, M., D’Elia, V., \& Palumbo, G. G. C. 2005, A\&A, 430, 39

Chary, R., \& Elbaz, D. 2001, ApJ, 556, 562

Coia, D., et al. 2005, A\&A, 431, 433

Cowie, L. L., \& Barger, A. J. 2008, ApJ, 686, 72

Croton, D. J., et al. 2006, MNRAS, 365, 11

Dale, D. A., \& Helou, G. 2002, ApJ, 576, 159

Davoodi, P., et al. 2006, MNRAS, 371, 1113

Eastman, J., Martini, P., Sivakoff, G., Kelson, D. D., Mulchaey, J. S., \& Tran, K.-V. 2007, ApJ, 664, L9

Fadda, D., Biviano, A., Marleau, F. R., Storrie-Lombardi, L. J., \& Durret, F. 2008, ApJ, 672, L9

Finn, R. A., Balogh, M. L., Zaritsky, D., Miller, C. J., \& Nichol, R. C. 2008, ApJ, 679,279

Fiore, F., et al. 2009, ApJ, 693, 447

Francis, P. J., Hewett, P. C., Foltz, C. B., Chaffee, F. H., Weymann, R. J., \& Morris, S. L. 1991, ApJ, 373, 465

Galametz, A., et al. 2009, ApJ, 694, 1309

Gallazzi, A., et al. 2009, ApJ, 690, 1883

Geach, J. E., et al. 2006, ApJ, 649, 661

Goto, T. 2005, MNRAS, 356, L6

Gunn, J. E., \& Gott, J. R. I. 1972, ApJ, 176, 1

Hopkins, A. M., \& Beacom, J. F. 2006, ApJ, 651, 142

Hopkins, P. F., Hernquist, L., Cox, T. J., \& Kereš, D. 2008, ApJS, 175, 356

Ilbert, O., et al. 2009, ApJ, 690, 1236

Johnston, D. E., et al. 2007, arXiv:0709.1159

Kennicutt, Jr., R. C. 1998, ARA\&A, 36, 189

Kinney, A. L., Calzetti, D., Bohlin, R. C., McQuade, K., Storchi-Bergmann, T., \& Schmitt, H. R. 1996, ApJ, 467, 38

Kocevski, D. D., Lubin, L. M., Lemaux, B. C., Gal, R., Fassnacht, C. D., Lin, R., \& Squires, G. K. 2008, arXiv:0809.2091

Koyama, Y., et al. 2008, MNRAS, 391, 1758
Krick, J. E., Surace, J. A., Thompson, D., Ashby, M. L. N., Hora, J. L., Gorjian, V., \& Yan, L. 2008, ApJ, 686, 918

Krick, J. E., et al. 2009, ApJS, submitted

Kronberger, T., Kapferer, W., Ferrari, C., Unterguggenberger, S., \& Schindler, S. 2008, A\&A, 481, 337

Lacy, M., et al. 2004, ApJS, 154, 166

Lilly, S. J., Le Fevre, O., Hammer, F., \& Crampton, D. 1996, ApJ, 460, L1

Lin, Y.-T., \& Mohr, J. J. 2007, ApJS, 170, 71

Lutz, D., Maiolino, R., Spoon, H. W. W., \& Moorwood, A. F. M. 2004, A\&A, 418, 465

Madau, P., Pozzetti, L., \& Dickinson, M. 1998, ApJ, 498, 106

Marcillac, D., Rigby, J. R., Rieke, G. H., \& Kelly, D. M. 2007, ApJ, 654, 825

Martini, P., Mulchaey, J. S., \& Kelson, D. D. 2007, ApJ, 664, 761

Mobasher, B., et al. 2004, ApJ, 600, L167

Moore, B., Katz, N., Lake, G., Dressler, A., \& Oemler, A. 1996, Nature, 379 613

Murakami, H., et al. 2007, PASJ, 59, 369

Negrello, M., et al. 2009, MNRAS, 394, 375

Onaka, T., et al. 2007, PASJ, 59, 401

Osmer, P. S. 2004, in Carnegie Observatories Centennial Symp., Coevolution of Black Holes and Galaxies, ed. L. C. Ho (Cambridge: Cambridge Univ. Press), 324

Pimbblet, K. A. 2003, PASA, 20, 294

Poggianti, B. M., et al. 2008, ApJ, 684, 888

Polletta, M., et al. 2007, ApJ, 663, 81

Press, W., Teukolsky, S., Vetterling, W., \& Flannery, B. 2007, Numerical Recipes: The Art of Scientific Computing (3rd ed.; Cambridge: Cambridge Univ. Press)

Rodighiero, G., et al. 2007, MNRAS, 376, 416

Rowan-Robinson, M., et al. 2008, MNRAS, 386, 697

Saintonge, A., Tran, K.-V. H., \& Holden, B. P. 2008, ApJ, 685, L113

Sajina, A., Lacy, M., \& Scott, D. 2005, ApJ, 621, 256

Salvato, M., et al. 2009, ApJ, 690, 1250

Staniszewski, Z., et al. 2008, arXiv:0810.1578

Stern, D., et al. 2005, ApJ, 631, 163

Surace, J. A., Shupe, D. L., Fang, F., Evans, T., Alexov, A., Frayer, D., \& Lonsdale, C. J. SWIRE Team 2005, BAAS, 1246

Ueda, Y., Akiyama, M., Ohta, K., \& Miyaji, T. 2003, ApJ, 598, 886

van Dokkum, P. G. 2005, AJ, 130, 2647 